\title{
Spectral and Energy Efficiencies in mmWave Cellular Networks for Optimal Utilization
}

\author{
Abdulbaset M. Hamed and Raveendra K. Rao \\ Faculty of Engineering, Department of Electrical and Computer Engineering, University of Western Ontario, London, \\ ON, Canada N6A 5B9 \\ Correspondence should be addressed to Raveendra K. Rao; rrao@uwo.ca
}

Received 29 September 2017; Revised 18 February 2018; Accepted 28 February 2018; Published 29 April 2018

Academic Editor: Ahmed M. Al-Samman

Copyright (C) 2018 Abdulbaset M. Hamed and Raveendra K. Rao. This is an open access article distributed under the Creative Commons Attribution License, which permits unrestricted use, distribution, and reproduction in any medium, provided the original work is properly cited.

\begin{abstract}
Millimeter wave (mmWave) spectrum has been proposed for use in commercial cellular networks to relieve the already severely congested microwave spectrum. Thus, the design of an efficient mmWave cellular network has gained considerable importance and has to take into account regulations imposed by government agencies with regard to global warming and sustainable development. In this paper, a dense mmWave hexagonal cellular network with each cell consisting of a number of smaller cells with their own Base Stations (BSs) is presented as a solution to meet the increasing demand for a variety of high data rate services and growing number of users of cellular networks. Since spectrum and power are critical resources in the design of such a network, a framework is presented that addresses efficient utilization of these resources in mmWave cellular networks in the 28 and $73 \mathrm{GHz}$ bands. These bands are already an integral part of well-known standards such as IEEE 802.15.3c, IEEE 802.11ad, and IEEE 802.16.1. In the analysis, a wellknown accurate mmWave channel model for Line of Sight (LOS) and Non-Line of Sight (NLOS) links is used. The cellular network is analyzed in terms of spectral efficiency, bit/s, energy efficiency, bit/J, area spectral efficiency, bit $/ \mathrm{s} / \mathrm{m}^{2}$, area energy efficiency, $\mathrm{bit} / \mathrm{J} / \mathrm{m}^{2}$, and network latency, s/bit. These efficiency metrics are illustrated, using Monte Carlo simulation, as a function of Signalto-Noise Ratio (SNR), channel model parameters, user distance from BS, and BS transmission power. The efficiency metrics for optimum deployment of cellular networks in 28 and $73 \mathrm{GHz}$ bands are identified. Results show that $73 \mathrm{GHz}$ band achieves better spectrum efficiency and the $28 \mathrm{GHz}$ band is superior in terms of energy efficiency. It is observed that while the latter band is expedient for indoor networks, the former band is appropriate for outdoor networks.
\end{abstract}

\section{Introduction}

The traffic in cellular networks has exponentially increased due to high demand for existing and a variety of new wireless services and growing number of users of networks. Therefore, it has become important to utilize the available bandwidth efficiently. Also, it has become mandatory, due to government regulations on greenhouse gas emissions, to pay careful attention to design of networks that are energy efficient as well. Thus, a key issue in the design and implementation of next generation of cellular networks is the joint optimization of energy and available spectral resources. The mmWave spectrum is known to offer tremendous bandwidth and can be utilized not only to meet the ever increasing demand for high data rate services, but also to accommodate a large number of users. This spectrum is also a serious contender for Ultra
High Definition Video (UHDV) transmission. Several wellknown network standards such as ECMA-387, IEEE 802.11ad, and IEEE $802.15 .3 \mathrm{c}$ are being redefined to fit this spectrum. Despite the massive bandwidth potential in the mmWave band, there exist a number of challenges: (i) modeling and characterization of propagation environment; (ii) design of antenna system; (iii) transceiver integration; (iv) digital signal processing technology.

The mmWave bands suffer from high path loss and hence techniques are required to enhance power received at the receiver. The modeling of propagation environment plays an important role in the design and analysis of communication systems required in cellular networks. In [1], characteristics of mmWave propagation environment are studied and it is observed that bands in this spectrum offer lower delay 
spread and it is possible to effectively suppress intersymbol interference. Wells [2] has shown that the 28 and $73 \mathrm{GHz}$ bands are the most natural choices for deployment in the mmWave spectrum. Also, the $28 \mathrm{GHz}$ band is being actively pursued for Local Multipoint Distribution System (LMDS) and is very attractive for use in mmWave cellular networks, given that this band occupies relatively lower range of frequencies within the mmWave spectrum.

In recent years, there have been many efforts to accurately model the mmWave channel for LOS and NLOS transmission links. The communication industry has devoted extensive resources to the development of accurate channel models. For instance, Samsung conducted channel measurements in the $28 \mathrm{GHz}$ band and came up with a model to fit ITU-R and FITU-R standards [3]. This model can be used for a mmWave communication link over a distance of up to 200 meters. In addition, Nokia, Huawei, and Deutsch Telekom have shown keen interest in the $73 \mathrm{GHz}$ band and have developed prototype hardware using the concept of Multiple Input Multiple Output (MIMO) system to support throughput of $20 \mathrm{Gbps}$. A channel model based on stochastic path loss has been studied for urban propagation environment in [4]. A spatial statistical model of mmWave channel has been developed as a function of channel parameters, including path loss, in [5]. This model has been derived based on real measurements at New York City in 28 and $78 \mathrm{GHz}$ bands for LOS and NLOS links.

A general framework has been proposed for evaluating coverage probability and rate performance in a mmWave cellular network in [6]. The short wavelength of mmWave signals can accommodate a very large number of antennas at the receiver and at the transmitter, which makes beamforming a key enabler technique in obtaining high antenna gain. Beamforming antenna systems for mmWave bands have been studied for $5 \mathrm{G}$ networks and a novel hybrid beamforming algorithm has been proposed for indoor and outdoor transmission links [7]. The design and characterization of mmWave antennas for wireless links are discussed in $[8,9]$.

Shannon's capacity limits and signal processing challenges for giga bit mmWave communication are discussed in [10]. Also, Madhow has addressed opportunities and challenges of utilizing MIMO in LOS mmWave communication systems. An overview of Impulse Radio-Ultra Wide Band (IR-UWB) technology for design of transmitters and receivers is presented in [11]. In [12], coverage and capacity of a dense mmWave network are analyzed using a theoretical model as a function of blockage probability and beamforming bandwidth. Also, the spectral efficiency (SE) (bits/s) of a mmWave network is investigated with LOS and NLOS links in 28 and $73 \mathrm{GHz}$ mmWave bands. Since minimal power consumption has received significance in recent years due to government regulations, it is important to address this issue for mmWave cellular networks. This issue has not received much attention in the literature. The efficiency metrics for LOS and NLOS links are evaluated for 28 and $73 \mathrm{GHz}$ bands in terms of maximum achievable throughput and maximum power efficiency in [13]. However, the work considered only path loss to represent the channel effects for transmission distances up to $200 \mathrm{~m}$. Tan et al. have investigated the achievable downlink spectral efficiency of multiuser mmWave cellular system considering both small- and large-scale fading effects in [14]. In [15], energy coverage probability was derived for mmWave transmission as a function of network density, beamforming bandwidth, and channel parameters.

The trade-off between energy efficiency and spectral efficiency is examined for different access networks in [16]. It is demonstrated that for best utilization of mmWave bands it is important to understand how to trade tolerable delay for low power. The delay-power trade-off is examined for design of an energy efficient cellular network in [17]. Levanen et al. have proposed technologies to reduce the delay in mmWave communication systems in [18]. The challenges of designing energy efficient mmWave systems are discussed in [19]. The energy efficiency (EE) (bit/J) metric is presented and evaluated for 28 and $73 \mathrm{GHz}$ mmWave bands for LOS and NLOS links. The investigation is presented as a function of BS transmission power, Signal-to-Noise Ratio (SNR), network parameters, and mmWave channel parameters. The spatial characteristics of cellular networks play a significant role in the performance of mmWave cellular networks. The area spectral efficiency (ASE) and the area energy efficiency (AEE) are important metrics that need to be considered in the design of mmWave networks.

Thus, the intent of this paper is to examine energy efficiency (EE), spectral efficiency (SE), network latency, area spectral efficiency (ASE), and area energy efficiency (AEE) of mmWave cellular network in 28 and $73 \mathrm{GHz}$ bands for LOS and NLOS links. The investigation is presented as a function of SNR, channel model parameters, network parameters, user distance from BS, and BS transmission power. This paper is organized as follows: In Section 2, mmWave cellular system is introduced including cellular network model, mmWave signal propagation and wireless channel models, and NR and SINR calculations. Section 3 presents evaluation of network performance metrics, spectral efficiency, energy efficiency, spatial spectral and energy efficiencies, network latency, and outage probability. Also, the evaluation of these efficiencies metrics is discussed. Simulation algorithm and the assumptions that have been made for our analysis are listed in Section 4. Moreover, the network and channel parameters are tabulated, and the numerical results with discussion are also presented. Finally, the work is concluded in Section 5.

\section{Millimeter Wave Cellular System}

The model of mmWave cellular system is shown in Figure 1. The macrocells are divided into small cells shown in the figure. The $J$ macrocells in the network are $(j=1, \ldots, J)$ using the same frequency and therefore create intercell interference. Each of the $j$ th cells comprises $I$ small cells each with its own BS $(i=1, \ldots, I)$ and uses the same frequency thereby causing intracell interference. It is assumed that each small cell has one BS and it serves $K$ Mobile Users (MUs) $(k=1, \ldots, K)$. The BSs are located at the center of small cells and connected to the gateway of the macrocell. The MUs are spatially distributed and each one is associated with a single BS at any time. 


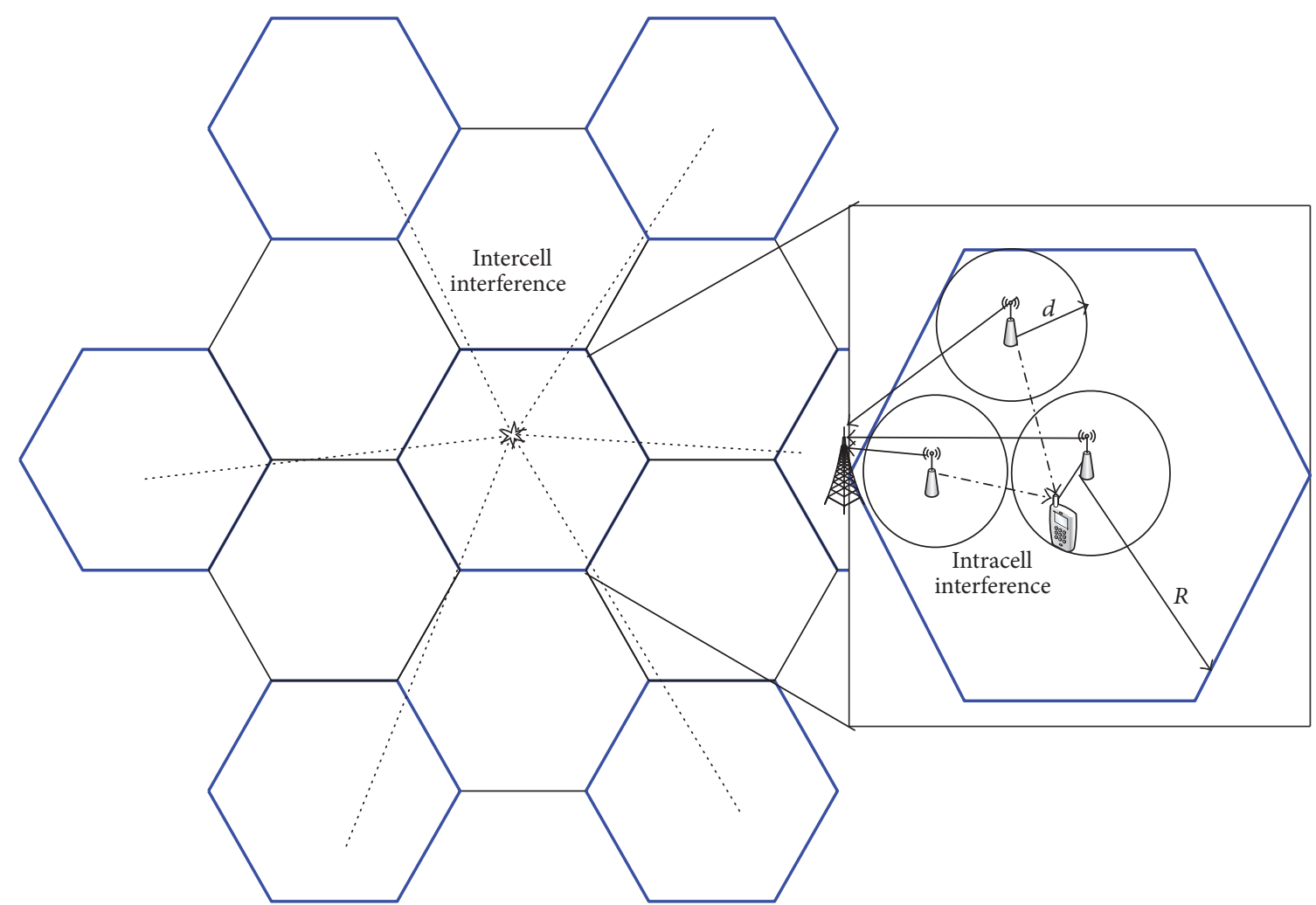

FIGURE 1: Model of mmWave cellular network in which the links “.-.-_" are for intracell interference, “...” for intercell interference, and "_-" for transmission between BSs where the cochannel macrocells are remarked in blue colour.

2.1. mmWave Wireless Channel Model. The mmWave signal is susceptible to high path loss, fading, noise, and interference; all these cause serious degradation in Signal-to-Noise Ratio (SNR) at the receiver leading to poor overall system performance. In general, mmWave communication channel can be represented by the double directional channel model given in [20] and is given by

$$
\begin{aligned}
\mathbf{H} & =\sum_{q=1}^{Q} \sum_{c=1}^{C} H_{q, c} e^{\phi_{q, c}} \delta\left(\tau-\tau_{q, c}\right) \delta\left(\phi_{R}-\phi_{\mathrm{AoA}, q, c}\right) \\
& \cdot \delta\left(\phi_{T}-\phi_{\mathrm{AoD}, q, c}\right) \delta\left(\theta_{R}-\theta_{\mathrm{ZoA}, q, c}\right) \delta\left(\theta_{T}-\theta_{\mathrm{ZoD}, q, c}\right),
\end{aligned}
$$

where $Q$ is the number of multipath components; $C$ is the number of rays within the cluster. Each ray is represented by path gain $H_{q, c}$, phase $\phi_{q, c}$, time-of-arrival (ToA) $\tau_{q, c}$, azimuth angle-of-arrival (AoA) $\phi_{\mathrm{AoA}, q, c}$, azimuth angle-of-departure (AoD) $\theta_{\mathrm{AoD}, q, c}$, zenith angle-of-arrival $(\mathrm{ZoA}) \theta_{\mathrm{ZoA}, q, c}$, and zenith angle-of-departure $(\mathrm{ZoD}) \theta_{\mathrm{ZoD}, q, c}$. The task of channel modeling is thus to find all these parameters for a mmWave communication channel. For 28 and $73 \mathrm{GHz}$ frequency bands, a model has been obtained to fit the LOS and NLOS transmission links. These bands were selected since they are the bands likely to be deployed in mmWave cellular network. According to this model [4], small-scale fading has a less impact on mmWave signal propagation, and hence the largescale fading effect is considered in measurements. In [5],
mmWave channel path loss effects are considered and a statistical model based on realistic measurements is presented for an urban environment, LOS and NLOS links, and is given by

$$
\begin{aligned}
L_{N / \operatorname{LOS}}[\mathrm{dB}]=\alpha+\beta 10 \log _{10}(r)+\xi[\mathrm{dB}] & \\
& \\
& \xi \sim \mathcal{N}\left(0, \sigma_{s}^{2}\right),
\end{aligned}
$$

where $L_{N / \text { LOS }}$ is the path loss in $\mathrm{dB}, r$ is the distance between transmitter and receiver in meters, $\alpha$ and $\beta$ are the least square fits of floating intercept and slope over the measured distances up to $200 \mathrm{~m}$, and $\sigma_{s}^{2}$ is the variance of the lognormal shadowing, $\xi$. In this paper, the channel model given by (2) [5] is used and the values of model parameters $\alpha, \beta$, and $\sigma_{s}^{2}$ are given in Table 1 .

2.2. Signal-to-Noise Ratio Calculation. The path loss model given by (2) is used to determine the received signal power at the MU and then used to estimate SNR of the $k$ th MU in the $i$ th BS. That is,

$$
\begin{aligned}
\mathrm{SNR}_{i, k[\mathrm{~dB}]} & \\
= & 10 \log _{10}\left|h_{i, k}\right|^{2}+P_{i[\mathrm{dBm}]}+G_{i[\mathrm{dBi}]}+G_{k[\mathrm{dBi}]} \\
& -L_{i, k[\mathrm{~dB}]}\left(r_{i, k}\right) \\
& -\left(\mathrm{KT}_{[\mathrm{dBm} / \mathrm{Hz}]}+10 \log _{10}\left(W_{m u}\right)+\mathrm{NF}_{[\mathrm{dB}]}\right),
\end{aligned}
$$


TABLE 1: Parameters of statistical channel model [5].

\begin{tabular}{lcccc}
\hline Path loss parameter & $\alpha$ & $\beta$ & $\sigma_{s}^{2}$ & \\
\hline $28 \mathrm{GHz}$ & & & 8.7 & \\
NLOS & 72 & 2.92 & 5.8 & \\
LOS & 61.4 & 2 & 5.0 & \\
$73 \mathrm{GHz}$ & & & 5.8 & \\
NLOS & 86.6 & 2.45 & 2 & \\
LOS & 69.8 & 2 & 5.8 \\
\hline
\end{tabular}

where $P_{i}$ is the $\mathrm{BS}_{i}$ transmission power, $\mathrm{KT}$ is the noise power density at the MU, and NF is the noise figure. The transmitter and receiver antenna gains, $G_{i}$ and $G_{k}$, are calculated using $G=20 \log _{10}(\pi l / \lambda)$ for the 28 and $73 \mathrm{GHz}$ frequency bands and antenna length of $l$. Assuming an independent Nakagami fading for each link, the small-scale fading component, $\left|h_{i, k}\right|^{2}$, can be modeled as a normalized Gamma random variable, that is, $\left|h_{i, k}\right|^{2} \sim \mathscr{G}(v, 1 / v)$ [15]. The small-scale fading is considered less severe in mmWave band [12], and hence $v$ is very large integer for LOS link, and $v=1$ when the link is NLOS. The path loss component $L_{i, k}$ can be calculated using (2).

2.3. Signal-to-Interference-Plus-Noise Ratio (SINR) Model. Based on the network model shown in Figure 1, for the $k$ th $\mathrm{MU}$ in the $i$ th small cell serving BS in the $j$ th macro cell, the interference consists of two components which are intracell interference and intercell interference. The first one is caused by the active BSs located in the same $j$ th cell which is more severe since it is close to the MU. While the second component is generated from the BSs in the other macro cells which is less intense. When the cochannel interference due to first tier macrocell is considered, the downlink aggregated interference in the $s$ small cell BS and $p$ macro cell for the $k$ th MU can be expressed as

$$
\begin{aligned}
\Lambda_{j, i, k}= & \sum_{\substack{i=1 \\
i \neq s}}^{I} P_{p, i} G_{p, i} G_{p, s, k}\left|h_{p, i, k}\right|^{2} L_{p, i, k}\left(r_{i, k}\right) \\
& +\sum_{\substack{j=1 \\
j \neq p}}^{I} \sum_{i=1}^{I} P_{j, i} G_{j, i} G_{j, i, k}\left|h_{j, i, k}\right|^{2} L_{j, i, k}\left(r_{j, i, k}\right) .
\end{aligned}
$$

Therefore, the SINR at the $\mathrm{MU}_{k}$ for $\mathrm{BS}_{i}$ is given by

$$
\operatorname{SINR}_{j, i, k[\mathrm{~dB}]}=\mathrm{SNR}_{i, k[\mathrm{~dB}]}-10 \log _{10}\left(\frac{\Lambda_{i, k}}{\sigma_{k}^{2}}+1\right),
$$

where $\sigma_{k}^{2}$ is the thermal noise power at the $k$ th MU. The distance between BS and MU (3) and (4) is <200 $\mathrm{m}$ and is a random variable with probability distribution function $f_{d}(r)=2 r / d^{2}$.

\section{Energy and Spectral Efficiency of mmWave Network}

Energy and spectrum are system resources that have to be appropriately traded to efficiently design a mmWave communication system. Minimal use of energy resources in the systems has become an important issue; however, efficient use of mmWave spectrum requires more power due to high path loss. Therefore, a balance between the use of energy and spectral resources of the system deserves a careful study. This is examined in this section for a mmWave cellular network. Analysis of energy and spectral efficiency metrics for such network is considered which can play an important role in the standardization of mmWave cellular network.

3.1. Network Spectral Efficiency. The downlink spectral efficiency quantifies the amount of data rate delivered by the BS to MUs over a certain bandwidth, $W_{k}$. This indicates how efficiently the mmWave spectrum is utilized. Spectral efficiency is bounded by Shannon's limit $W_{k} \log _{2}\left(1+\mathrm{SNR}_{k}\right)$ [21] for a given SNR. For the case of mmWave network SNR is replaced by SINR. Thus, spectral efficiency of the network, depicted in Figure 1, can be evaluated using

$$
\begin{aligned}
& \eta_{\mathrm{SE}}=\sum^{J} \sum^{I} \sum^{K} W_{k} \mathbb{E}_{r}\left[\mathbb{E}_{h}\left[\log _{2}\left(1+\operatorname{SINR}_{j, i, k}\right)\right]\right] \\
& \cdot\left(1-P_{\text {out }, j}(R)\right),(\text { bit } / \mathrm{s}),
\end{aligned}
$$

where SINR can be obtained from (5), and $W_{k}$ is the allocated mmWave bandwidth for the MUs. $\mathbb{E}_{r}$ and $\mathbb{E}_{h}$ are the expectation values taken for over MU's random location and the small-scale fading effect, respectively. The outage probability within a communication range $R, P_{\text {out, } j}$, is given by [22]

$$
P_{\text {out }, j}(R)=\exp \left(-\frac{2 \pi \rho_{j}}{\gamma_{j}^{2}}\left(1-\left(1+\gamma_{j} R\right) e^{-\gamma_{j} R}\right)\right),
$$

where $\gamma$ is the parameter that depends on the propagation environment density, and $\rho$ is the BS density. The outage probability is plotted as a function of $\gamma$ and $R$ for a given $\rho=$ $10^{-4}$ in Figure 2 . It is observed that $P_{\text {out }}$ is nearly constant for $R>300 \mathrm{~m}$. Since the proposed network dimensions are large, with macro cell radius $R \geqslant 500 \mathrm{~m}$, the outage probability can be approximated by $P_{\text {out }, j}=e^{-2 \pi \rho_{j} / \gamma_{j}^{2}}$.

3.2. Network Energy Efficiency. The energy efficiency is defined as a ratio between what the system delivers to what it consumes, and hence the efficiency function can be described as $f(x) / x$, where $x \in[0, X]$ indicates the system's resources constrained by $X$ [23]. In this paper, $f(x)$ represents the 
TABLE 2: Parameters used in the simulation of cellular network

\begin{tabular}{lcc}
\hline Symbol & Descriptions & Value \\
\hline$W_{k}$ & User bandwidth & $0.1 \mathrm{GHz}$ \\
$P_{i}$ & BS transmission power & $-10 \mathrm{dBm}-40 \mathrm{dBm}$ \\
$\mathrm{KT}$ & Noise power density & $-174 \mathrm{dBm} / \mathrm{Hz}$ \\
$\mathrm{NF}$ & Noise figure & $6 \mathrm{~dB}$ \\
$d$ & Maximum BS serving distance & $100 \mathrm{~m}$ \\
$R$ & Macro cell radius & $500 \mathrm{~m}$ \\
$J$ & Maximum number of cells sharing the spectrum & 7 \\
$I$ & Maximum number of BSs in the macro cell & uniform $\sim 1 / \rho$ \\
$K$ & Number of simultaneous users in BSs & 20 \\
$\rho$ & BS density & $\left(\frac{d}{R}\right)^{2}$ \\
$\gamma$ & Propagation environment density & $10^{-2}$ \\
$l_{t}$ & Transmitter antenna length & $0.1 \mathrm{~m}$ \\
$l_{r}$ & Receiver antenna length & $0.005 \mathrm{~m}$ \\
\hline
\end{tabular}

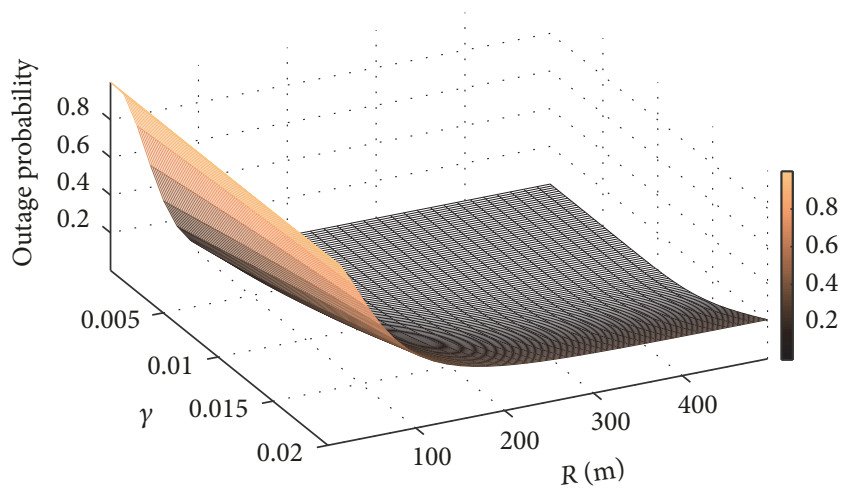

FIGURE 2: Outage probability as a function of $\gamma$ and $R$ for $\rho=10^{-4}$.

overall downlink data rate efficiency that can be reliably transmitted by a mmWave communication system, and $x$ denotes the Signal-to-Noise Ratio, which is a function of BS's transmission power, noise, and channel effects. For the mmWave network, the energy efficiency can be formulated as

$$
\begin{aligned}
\eta_{\mathrm{EE}} & =\sum^{J} \sum^{I} \sum^{K} W_{k} \mathbb{E}_{r}\left[\mathbb{E}_{h}\left[\frac{\log _{2}\left(1+\mathrm{SINR}_{j, i, k}\right)}{\mathrm{SNR}_{j, i, k}}\right]\right] \\
\cdot\left(1-P_{\text {out }, j}(R)\right),(\mathrm{bit} / \mathrm{J}) &
\end{aligned}
$$

where SNR and SINR can be determined using (3) and (5), respectively. The energy efficiency indicates the amount of data rate that can be delivered per unit of SNR per Hertz of bandwidth. The efficiency metrics are useful in the standardization of mmWave cellular networks, as spectral efficiency provides the maximum rate for each frequency band and transmission range and energy efficiency gives insight into how to utilize the energy resources in cells as a function of data rate.
3.3. Spatial Spectral and Energy Efficiency. Spatial characteristics of mmWave network are crucial in the evaluation of system performance. For example, the propagation loss is highly affected by network dimensions, and hence SNR and SINR are also impacted. Therefore, studying the spectral and energy efficiencies is not enough for efficient design of mmWave networks. The spatial spectral and energy efficiencies are better metrics for design of networks [24]. Accordingly, these metrics are examined for mmWave networks. These metrics are evaluated for macrocell as a sum of each small cell spatial efficiency in which the serving distance, $r$, determines the cells' area.

\section{Numerical Results}

Monte Carlo simulations have been performed in MATLAB environment to evaluate spectral, energy and spatial spectral and energy efficiencies for the 28 and $73 \mathrm{GHz}$ mmWave bands. The efficiency metrics are investigated in terms of BS transmission power, BS serving distance, and SNR. The steps used in the simulation are given below:

(1) The path loss for 28 and $73 \mathrm{GHz}$ bands is calculated for LOS and NLOS using (2) for each value of $P$ and $r$, and the model parameters are given in Table 1 .

(2) SNR is computed using (3), in which small-scale fading is modelled using Gamma distribution, $|h|^{2} \sim$ $\mathscr{C}(v, 1 / v)$, and (3) parameters are given in Table 2.

(3) For the network model, Figure 1, the aggregated intercell and intracell interferences are obtained using (4).

(4) The SINR is determined (5) for $\sigma_{k}^{2}=\mathrm{KTW}_{k}$.

(5) Spectral and energy efficiency metrics are computed using (6) and (8) with $P_{\text {out }}$ calculated using (7); see Figure 2.

(6) The spatial spectral and energy efficiency metrics are computed using $\sum\left(\eta_{\mathrm{SE}, j, i, k} / A_{j, i}\right)$ and $\sum\left(\eta_{\mathrm{EE}, j, i, k} / A_{j, i}\right)$, 
respectively, with $A_{j, i}=\pi d_{j, i}^{2}$ being the coverage area of each small cell BS [25].

The following assumptions are made in Monte Carlo simulation.

(1) Channel fading coefficients, $h_{i, j, k}$, in (3) and (4) are independent and identical distributed random variables.

(2) Transmission power, $P_{i}$, in (3), is identical for all small cell BSs.

(3) Antenna gain, $G_{i}$, is equal for all BSs that are assumed to be same, and antenna gain, $G_{k}$, for all MUs is assumed to be identical.

(4) Intercell interference is based on only the first tier of the macrocells.

(5) Intercell distance, $r_{i, j, k}=3 R+d$, and the intracell distance, $r_{i, k}=2 d$, represent the worst MUs in small cells.

(6) Macro cell radius of $R=500 \mathrm{~m}$ is assumed and the maximum serving distance of a small cell BS is set to $d=100 \mathrm{~m}$.

The cellular network parameters used in the simulations are given in Table 2. Simulation and numerical process of this work are summarized in the flow chart shown in Figure 3.

4.1. Spectral Efficiency. The spectral efficiency of the network is evaluated in terms of achievable data rate for a specific allocated bandwidth to MU. This efficiency metric (bit/s) is plotted in Figure 4 as a function of maximum serving distances for LOS and NLOS links in 28 and $73 \mathrm{GHz}$ bands with transmission power fixed at $10 \mathrm{dBm}$. It is observed that the maximum achievable data rate in the network can reach $1 \mathrm{Tbit} / \mathrm{s}$ as $d$ is diminished. In this case, the macrocell will have a large number of small cells and intracell interference is increased. Also, it is noted that the spectral efficiency slightly deteriorates for LOS link while for NLOS link it decreases rapidly to $0.15 \mathrm{G}$ bit $/ \mathrm{s}$ for $d=100 \mathrm{~m}$. The spectral efficiency is also investigated as a function of BS power used for transmission for a specific value of serving distance, $d=$ $45 \mathrm{~m}$. Figure 5 demonstrates that the spectral efficiency is enhanced as power transmitted by the BS is increased. It is observed that there is no significant improvement in the spectral efficiency when the transmission power is above $40 \mathrm{dBm}$. This indicates that pumping more power is waste of BS power resource and hence impacts the energy efficiency. Thus, for optimum mmWave band utilization, transmission power over $40 \mathrm{dBm}$ is not required. The figure also illustrates the superiority of high frequency band, $73 \mathrm{GHz}$, for the LOS link.

For high power transmission, the achieved spectral efficiencies of the two transmission bands are above $1 \mathrm{Tbit} / \mathrm{s}$. Since the SNR at MUs is a function of the BS transmission power, receiver noise, and channel effects, the spectral efficiency for the $73 \mathrm{GHz}$ band for LOS link is presented in Figure 6 as a function of serving distance and SNR. It is noted that the spectral efficiency is an increasing function of SNR which indicates the mmWave system can overcome the noise and channel impairments. The spectral efficiency of mmWave cellular networks has been investigated in $[14,26]$ and the numerical results presented in these works are consistent with the results illustrated in Figure 5. However, the spectral efficiency results of this paper are based on real channel measurements.

4.2. Energy Efficiency. Energy efficiency metric is a "green communication" indicator, since the transmitted power by the BS plays an important role in its determination. The energy efficiency metric is a function of the data rate over the mmWave links and SNR at MUs. The energy efficiency is examined as a function of BS serving distance, the transmission power, and the SNR. The metric is plotted as a function of transmission power for two mmWave bands and links in Figure 7. It is observed that the $73 \mathrm{GHz}$ band is less energy efficient compared to the $28 \mathrm{GHz}$ band. The energy efficiency is degraded as the transmission power is increased. Figure 8 shows a comparison of energy efficiency between the two frequency bands for LOS transmission link as a function of $d$ and SNR. It is observed that $28 \mathrm{GHz}$ band, Figure 8(b), attains higher energy efficiency than the $73 \mathrm{GHz}$ band, Figure 8(a). In fact, the SNR for LOS transmission at $73 \mathrm{GHz}$ band is greater than the SNR for NLOS link at the $28 \mathrm{GHz}$ band. Also, it is noted that the efficiency improves up to certain serving distance, $d=30 \mathrm{~m}$, and then becomes almost constant for $d>30 \mathrm{~m}$. It is noted that trade-off between serving distance and the transmission power has to be considered in dimensioning of the network and for optimum utilization for the two mmWave bands. Energy efficiency of mmWave cellular network has been examined in terms of number of users and BS antenna size for full-duplex relaying backhaul small cell mmWave networks in [19] for $28 \mathrm{GHz}$ and $60 \mathrm{GHz}$ bands. The results show an increasing function of the transmitted power which contradicts the energy efficiency concept illustrated in Figure 7. Also, this metric has been investigated in terms of propagation environment parameters and BS density for heterogeneous mmWave network overlaid by small cell BSs in [22]. The results are consistent with the results illustrated in Figure 8.

4.3. Network Latency. Delay is critical metric for real time applications in mmWave networks. Thus, it is important to address the network delay as a function of network parameters. The delay in mmWave networks is due to several components such as radio access network, backhaul, core network, and data centers. In this paper, we consider the transmission delay between $\mathrm{MU}$ and BS. For a given user bandwidth and information quantity $Q_{k}$, the transmission delay $D$ is given by $D=Q_{k} / C$ [27]. For the network shown in Figure 1, the delay-per-bit per user can be expressed as

$$
\begin{aligned}
D_{k} & =\frac{Q_{k}}{W_{k} \mathbb{E}_{r}\left[\mathbb{E}_{h}\left[\log _{2}\left(1+\operatorname{SINR}_{j, i, k}\right)\right]\right]\left(1-P_{\text {out }, j}(R)\right)},
\end{aligned}
$$

where $Q_{k}=1$ and $\operatorname{SINR}_{j, i, k}$ is given by (5). The tradeoff among latency and efficiency metrics of the mmWave 


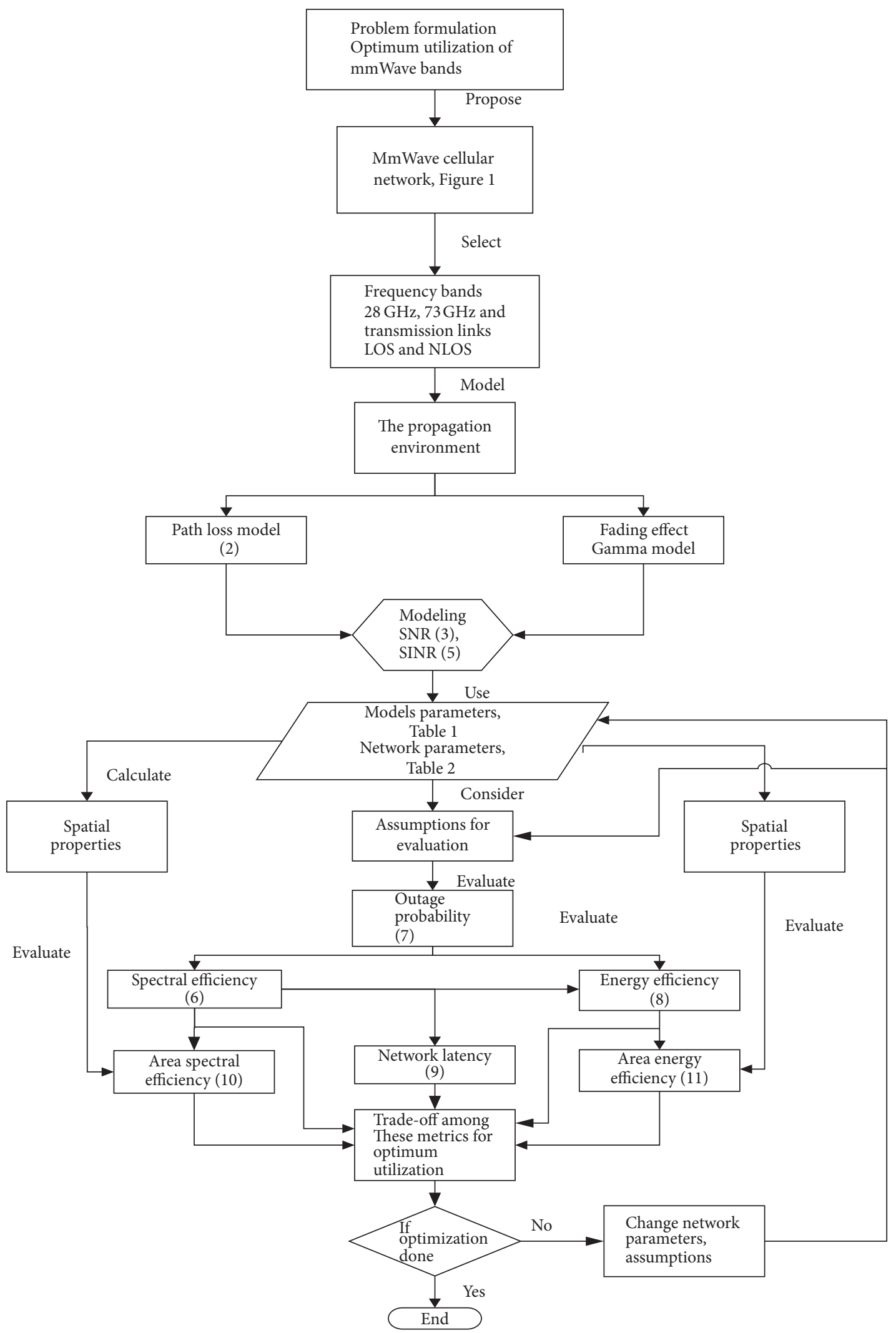

FIGURE 3: Flow chart used for evaluation of trade-off between spectral and energy efficiencies for optimum utilization in mmWave network. 


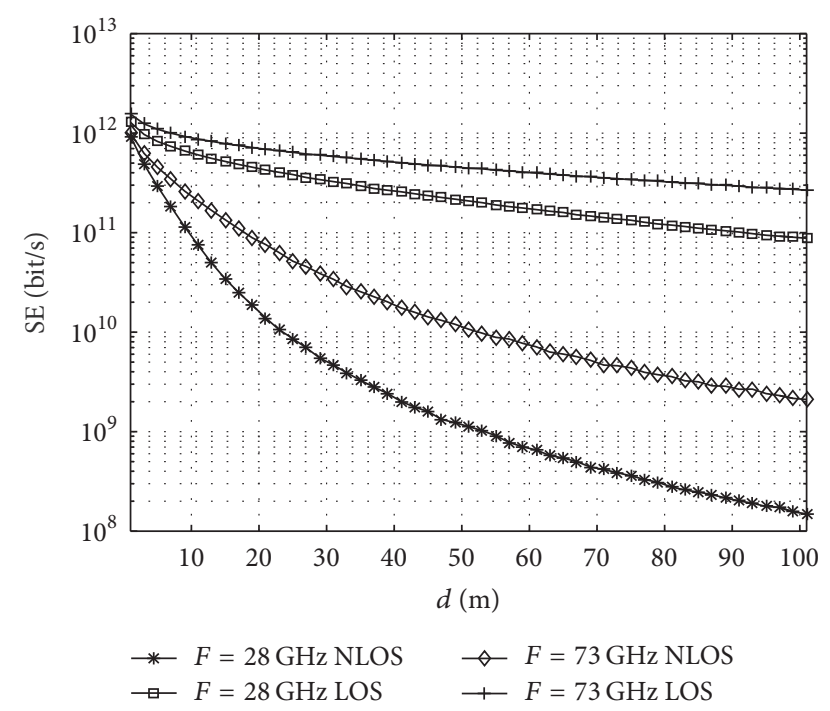

FIGURE 4: Spectral efficiency of $28 \mathrm{GHz}$ and $73 \mathrm{GHz}$ mmWave bands for NLOS and LOS links as a function of the maximum serving distance.

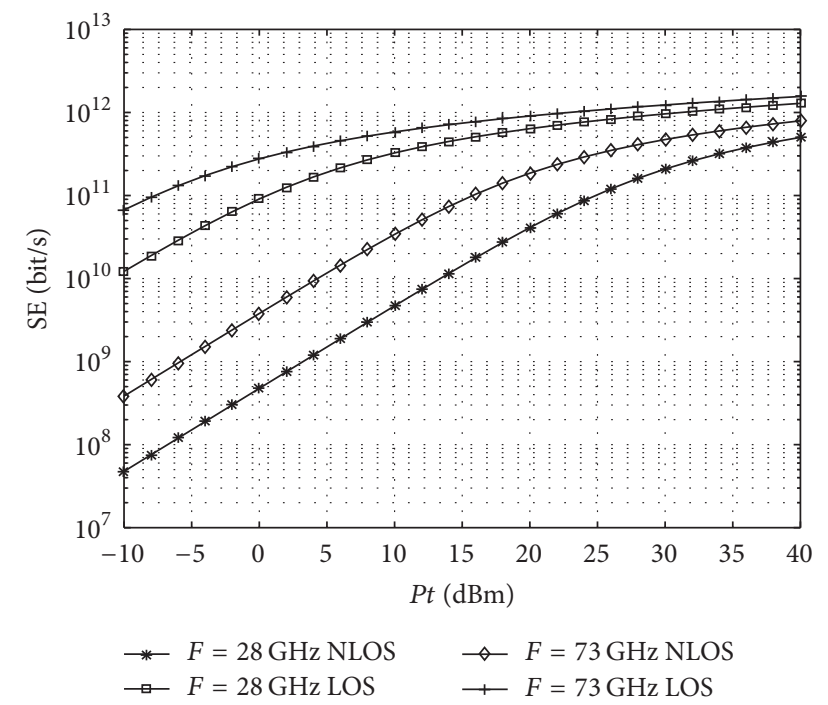

FIGURE 5: Spectral efficiency of $28 \mathrm{GHz}$ and $73 \mathrm{GHz}$ mmWave bands for NLOS and LOS links as a function of transmission power.

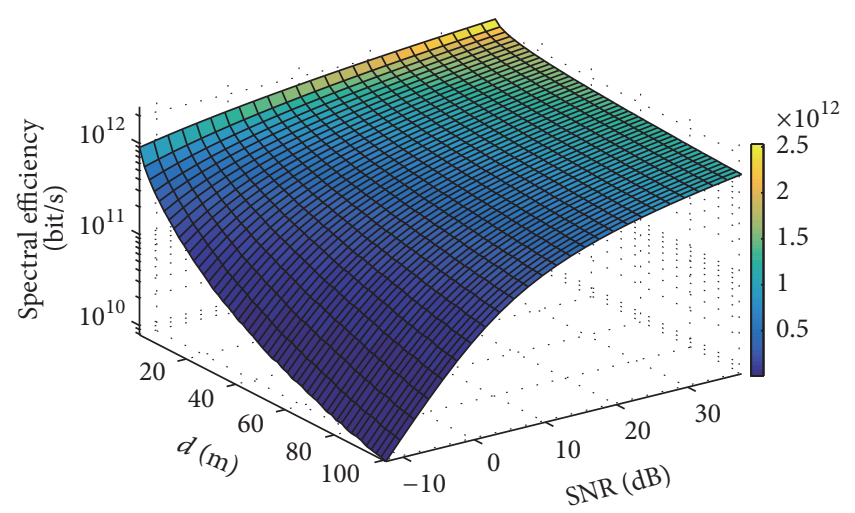

FIGURE 6: Spectral efficiency of $73 \mathrm{GHz}$ band LOS link as a function of $d$ and SNR.

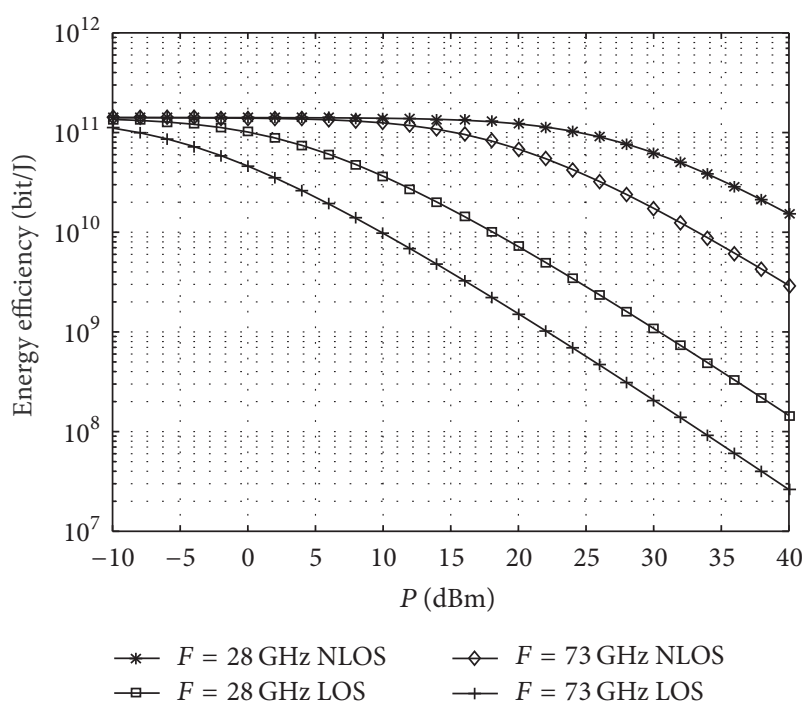

FIGURE 7: Energy efficiency of $28 \mathrm{GHz}$ and $73 \mathrm{GHz}$ mmWave bands for NLOS and LOS links as a function of BS power.

network can be discussed in terms of delay-per-bit and energy efficiency. Delay-per-bit is obtained by simulating (9) using channel parameters given in Table 1 and network parameters given in Table 2. The delay is plotted as a function of BS power and user distance in Figures 9(a) and 9(b), respectively. These figures show that $73 \mathrm{GHz}$ band for LOS link achieves less latency than the $28 \mathrm{GHz}$ band for NLOS link. Figure 9(a) shows that the delay decreases as the BS power increases. For example, the network latency is less than $1 \mathrm{~ns} / \mathrm{bit}$ when the BS power is greater than $20 \mathrm{dBm}$. The energy efficiency is highly impacted for such large values of BS power as shown in Figure 7. In terms of user distance, the network latency increases as the distance increases as is clear from Figure 9(b). In contrast the spectral efficiency is reduced as a function of user distance which indicates that the two metrics are in conflict. Therefore, trade-offs among network latency, energy efficiency, and spectral efficiency metrics must be considered for optimum utilization of the mmWave bands.

4.4. Area Spectral Efficiency. The area spectral efficiency (ASE) is defined as the ratio of the average spectral efficiency to the utilized area for a given frequency band. For the network in Figure 1, The ASE is calculated as the sum of the spectral efficiencies of each small cell divided by its serving area and is given by

$$
\sum \frac{\eta_{\mathrm{SE}, j, i, k}}{A_{j, i}}
$$

This ASE metric links the network spectral efficiency and the service area and is investigated as a function of $P, d$, and SNR. In Figure 10(a), ASE is plotted in terms of $d$. It is noted that ASE is a decreasing function of the distance since the area is proportional to $d^{2}$. Also, ASE is plotted in terms of $d$ and SNR in Figure 10(b) which depicts how both factors affect the ASE. It is clear that for large coverage area the network requires more BS power to achieve acceptable ASE. 


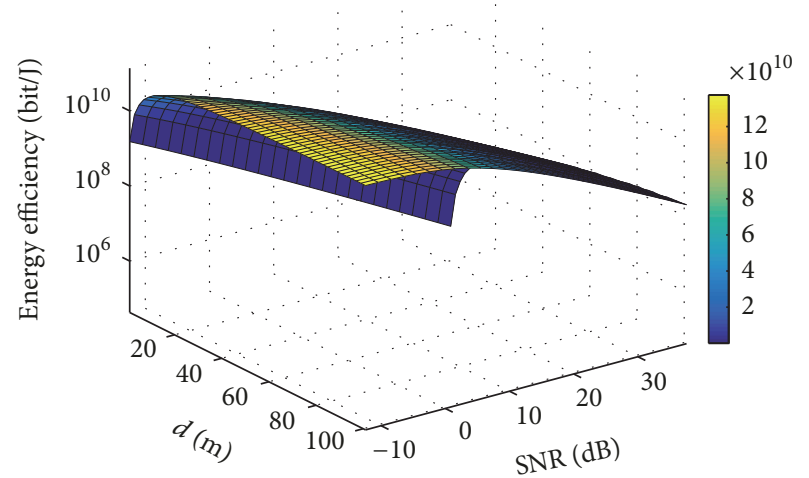

(a) $73 \mathrm{GHz}$ bands for LOS

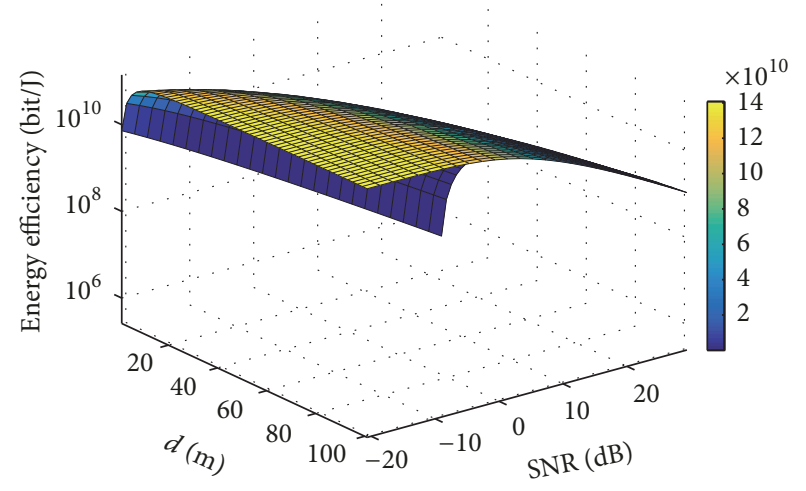

(b) $28 \mathrm{GHz}$ bands for LOS

FIGURE 8: Energy efficiency of 73 and $28 \mathrm{GHz}$ bands for LOS link as a function of $d$ and SNR.

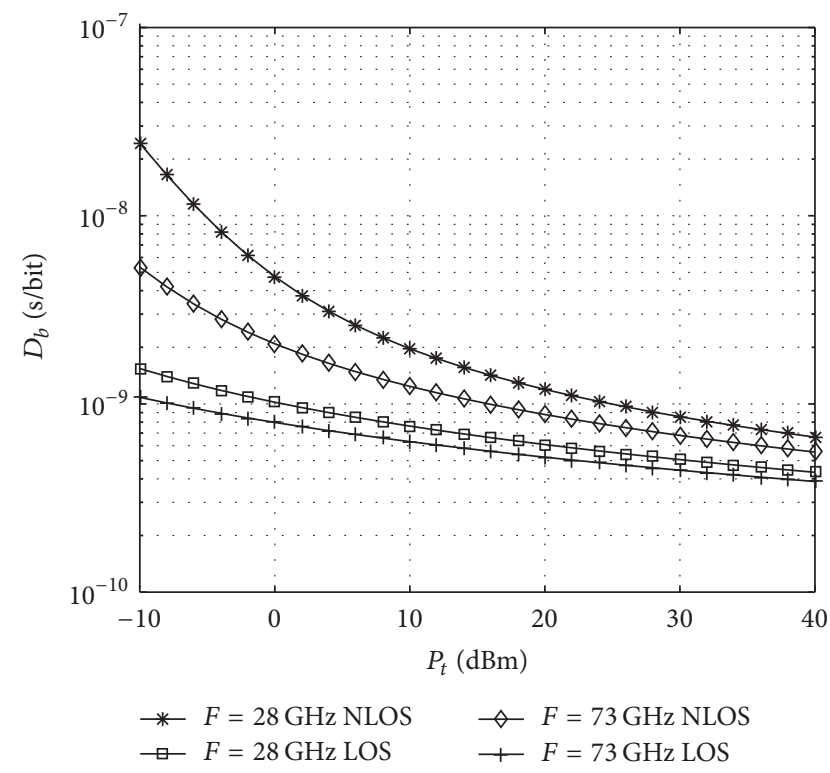

(a) Delay-per-bit as a function of transmitted power $p_{t}$

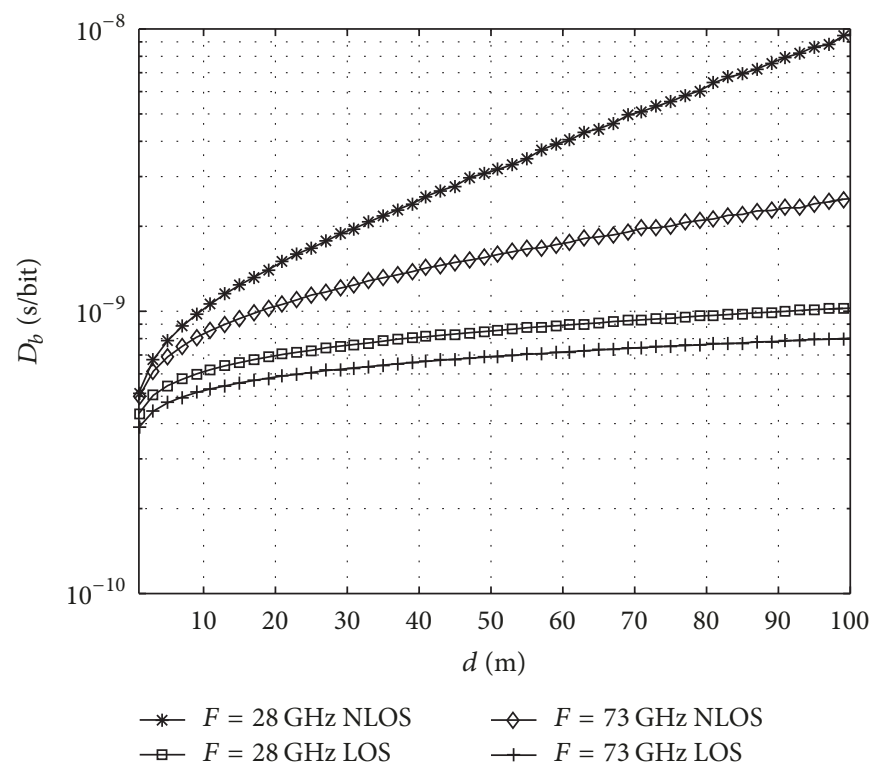

(b) Delay-per-bit as a function of user distance $d$

FIGURE 9: Delay-per-bit for 73 and $28 \mathrm{GHz}$ bands for LOS and NLOS links.

4.5. Area Energy Efficiency (AEE). The AEE is used to characterize the area data rate per unit $\mathrm{BS}$ power expenditure and can be defined as

$$
\mathrm{AEE}=\sum \frac{\eta_{\mathrm{EE}, j, i, k}}{A_{j, i}},
$$

where $\eta_{\mathrm{EE}, j, i, k}$ is given by (8) and $A_{j, i}=\pi d_{j, i}^{2}$. This metric provides insight into the deployment and implementation challenges for optimum mmWave band utilization. The AEE is evaluated as a function of BS power and is shown in Figure 11(b). The metric severely deteriorates for high values of BS power; however, it maintains a certain level of performance at lower values of power. Also, it is observed that AEE slightly decreases as the radius of small cell increases as shown in Figure 11(a).

From the above results, it is clear that there is always a conflict between spectral efficiency and energy efficiency in terms of power transmission. The optimum utilization of mmWave bands requires trade-off between these two performance metrics. The $28 \mathrm{GHz}$ band achieves higher energy efficiency for a wide range of power transmission level while it provides acceptable spectral efficiency for $P_{t}>20 \mathrm{dBm}$. On the other hand, the $73 \mathrm{GHz}$ is superior in terms of spectral efficiency; however, it performs poorly with respect to energy efficiency. Regarding the transmission environment, it can be said that the $28 \mathrm{GHz}$ band is more advantageous than the $73 \mathrm{GHz}$ band for NLOS links such as indoor networks. However, the $73 \mathrm{GHz}$ bands are more suitable for LOS link such as outdoor mmWave links.

\section{Conclusions}

In this paper, mmWave cellular network is considered for indoor and outdoor applications in which the macrocells are 


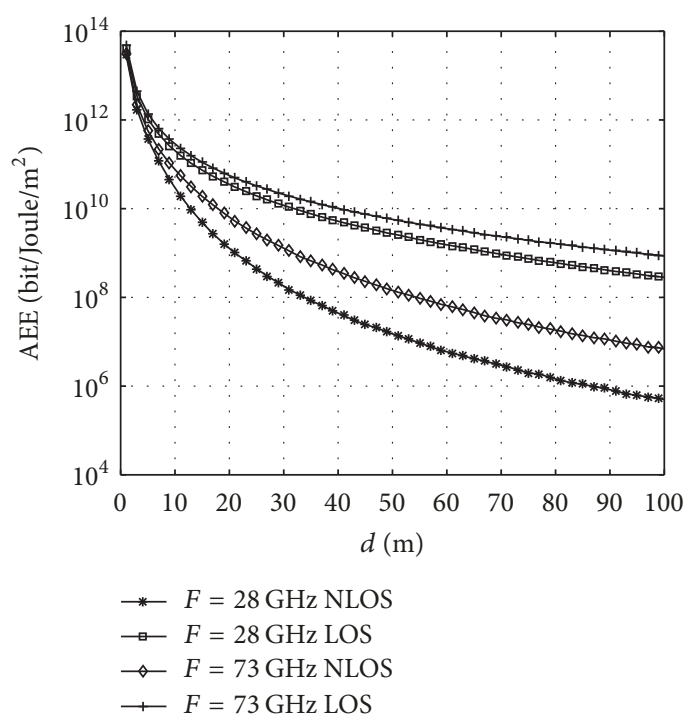

(a) ASE of 73 and $28 \mathrm{GHz}$ bands for LOS and NLOS links as a function of BS serving distance

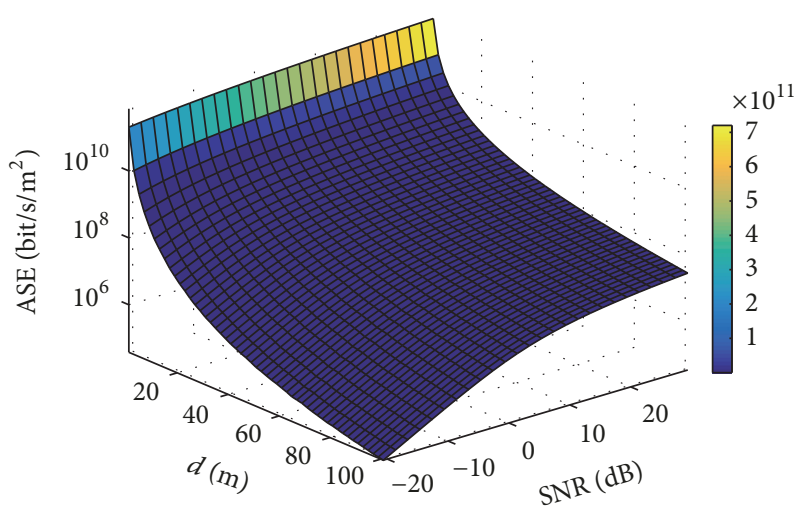

(b) ASE of $73 \mathrm{GHz}$ band LOS as function of $d$ and SNR

FIGURE 10: Area spectral efficiency of mmWave bands.

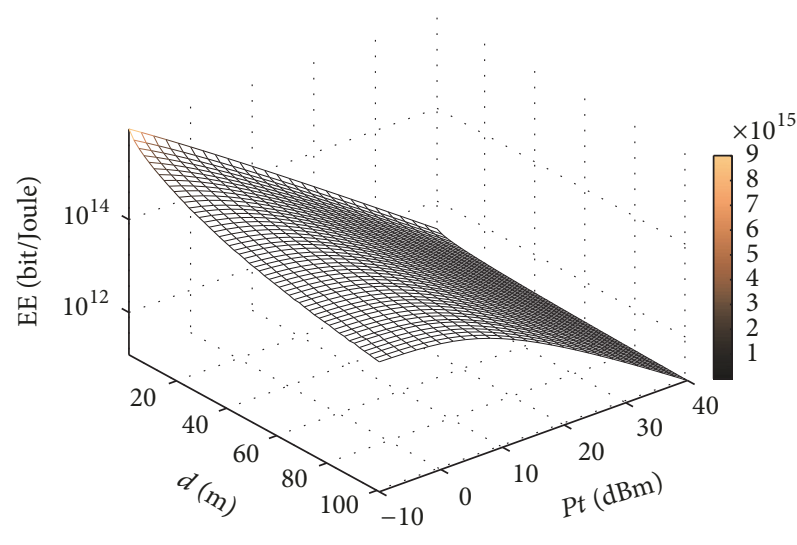

(a) AEE 73 GHZ band LOS

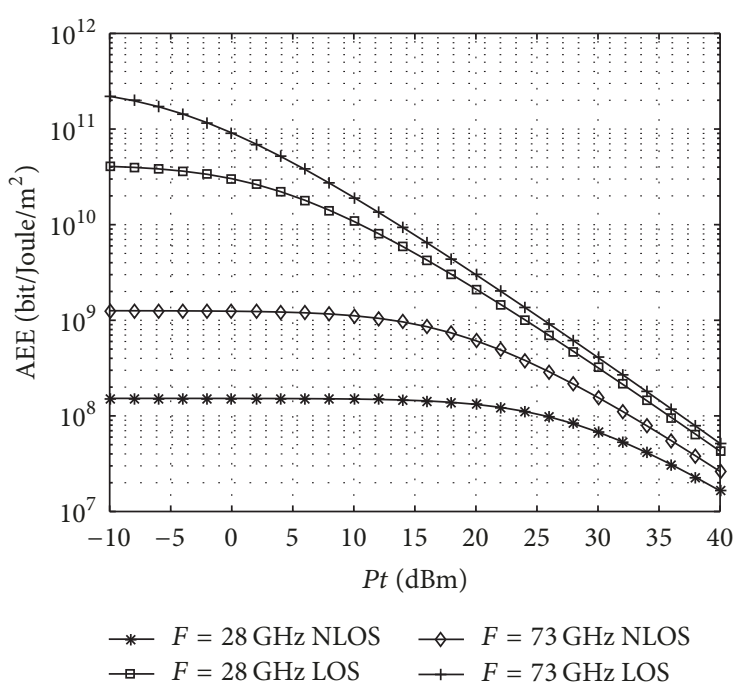

(b) AEE of 73 and 28 GHZ bands for LOS and NLOS

Figure 11: Area energy efficiency of mmWave bands as a function of $d$ and $P$.

overlaid with multiple small cells. A channel model based on real measurements available in literature for LOS and NLOS transmission links was used to derive expressions for SNR and SINR in network considering intercell and intracell interference components. The paper presented a framework for analysis of spectral and energy efficiency metrics of the network for optimum utilization of network resources. The framework also included an investigation of spectral efficiency, energy efficiency, and spatial performance metrics. The analysis was done using Monte Carlo simulation considering the effects of interference, the path loss, and small-scale fading. The investigation of metrics has been carried out as a function of BS power, SNR, mmWave network parameters, and channel model parameters. The results show that the spectral efficiency is always in conflict with the energy efficiency with regard to BS power and the maximum BS serving distance. The $28 \mathrm{GHz}$ band can be expedient for NLOS links in the network, and the $73 \mathrm{GHz}$ band is more appropriate for LOS links.

\section{Conflicts of Interest}

The authors declare that they have no conflicts of interest. 


\section{Acknowledgments}

The first author would like to acknowledge University of Tripoli, Libya, and the Libyan Ministry of Education for their support and scholarship.

\section{References}

[1] Y. Li, P. Wang, F. Chen, G. Wang, and Y. Liu, "Simulation and Analysis of Millimeter-Wave Propagation Characteristics in Complex Office Environment," Journal of Computer and Communications, vol. 03, no. 03, pp. 56-60, 2015.

[2] J. Wells, "Faster than fiber: The future of multi-G/s wireless," IEEE Microwave Magazine, vol. 10, no. 3, pp. 104-112, 2009.

[3] J. Ko, Y.-S. Noh, Y.-C. Kim et al., " $28 \mathrm{GHz}$ millimeter-wave measurements and models for signal attenuation in vegetated areas," in Proceedings of the 11th European Conference on Antennas and Propagation, EUCAP 2017, pp. 1808-1812, France, March 2017.

[4] T. Bai, V. Desai, and R. W. Heath Jr., "Millimeter wave cellular channel models for system evaluation," in Proceedings of the 2014 International Conference on Computing, Networking and Communications, ICNC 2014, pp. 178-182, USA, February 2014.

[5] M. R. Akdeniz, Y. Liu, M. K. Samimi et al., "Millimeter wave channel modeling and cellular capacity evaluation," IEEE Journal on Selected Areas in Communications, vol. 32, no. 6, pp. 11641179, 2014.

[6] L. Zhao, J. Cai, and H. Zhang, "Radio-efficient adaptive modulation and coding: Green communication perspective," in Proceedings of the 2011 IEEE 73rd Vehicular Technology Conference, VTC2011-Spring, Hungary, May 2011.

[7] W. Roh, J.-Y. Seol, J. Park et al., "Millimeter-wave beamforming as an enabling technology for $5 \mathrm{G}$ cellular communications: theoretical feasibility and prototype results," IEEE Communications Magazine, vol. 52, no. 2, pp. 106-113, 2014.

[8] X. Wu, G. V. Eleftheriades, and T. E. Van Deventer-Perkins, "Design and characterization of single- and multiple-beam MM-wave circularly polarized substrate lens antennas for wireless communications," IEEE Transactions on Microwave Theory and Techniques, vol. 49, no. 3, pp. 431-441, 2001.

[9] Y. P. Zhang and D. Liu, "Antenna-on-chip and antenna-inpackage solutions to highly integrated millimeter-wave devices for wireless communications," IEEE Transactions on Antennas and Propagation, vol. 57, no. 10, pp. 2830-2841, 2009.

[10] U. Madhow, "MultiGigabit millimeter wave communication: System concepts and challenges," in Proceedings of the 2008 Information Theory and Applications Workshop - ITA, pp. 193196, USA, February 2008.

[11] C. Stallo, E. Cianca, S. Mukherjee, T. Rossi, M. de Sanctis, and M. Ruggieri, "UWB for multi-gigabit/s communications beyond 60 GHz," Telecommunication Systems, vol. 52, no. 1, pp. 161-181, 2013.

[12] T. Bai, A. Alkhateeb, and R. W. Heath, "Coverage and capacity of millimeter-wave cellular networks," IEEE Communications Magazine, vol. 52, no. 9, pp. 70-77, 2014.

[13] A. M. Hamed and R. K. Rao, "Evaluation of capacity and power efficiency in millimeter-wave bands," in Proceedings of the 2016 International Symposium on Performance Evaluation of Computer and Telecommunication Systems, SPECTS 2016, Canada, July 2016.

[14] W. Tan, P. J. Smith, H. A. Suraweera, M. Matthaiou, and S. Jin, "Spectral efficiency of multi-user mmwave systems with uniform linear arrays and MRT,' in Proceedings of the 83rd IEEE Vehicular Technology Conference, VTC Spring 2016, China, May 2016.

[15] T. A. Khan, A. Alkhateeb, and R. W. Heath, "Energy coverage in millimeter wave energy harvesting networks," in Proceedings of the IEEE Globecom Workshops, GC Wkshps 2015, USA, December 2015.

[16] A. Mesodiakaki, F. Adelantado, A. Antonopoulos, L. Alonso, and C. Verikoukis, "Energy and spectrum efficient user association in 5G heterogeneous networks," in Proceedings of the 27th IEEE Annual International Symposium on Personal, Indoor, and Mobile Radio Communications, PIMRC 2016, Spain, September 2016.

[17] Y. Chen, S. Zhang, S. Xu, and G. Y. Li, "Fundamental trade-offs on green wireless networks," IEEE Communications Magazine, vol. 49, no. 6, pp. 30-37, 2011.

[18] T. Levanen, J. Pirskanen, and M. Valkama, "Radio interface design for ultra-low latency millimeter-wave communications in 5G Era," in Proceedings of the 2014 IEEE Globecom Workshops, GC Wkshps 2014, pp. 1420-1426, USA, December 2014.

[19] Z. Wei, X. Zhu, S. Sun, Y. Huang, A. Al-Tahmeesschi, and Y. Jiang, "Energy-Efficiency of Millimeter-Wave Full-Duplex Relaying Systems: Challenges and Solutions," IEEE Access, vol. 4, pp. 4848-4860, 2016.

[20] M. Steinbauer, A. F. Molisch, and E. Bonek, "The doubledirectional radio channel," IEEE Antennas and Propagation Magazine, vol. 43, no. 4, pp. 51-63, 2001.

[21] C. E. Shannon, "A mathematical theory of communication," Bibliometrics, vol. 5, no. 1, pp. 3-55, 2001.

[22] J. Choi, "Energy efficiency of a heterogeneous network using millimeter-wave small-cell base stations," in Proceedings of the 26th IEEE Annual International Symposium on Personal, Indoor, and Mobile Radio Communications, PIMRC 2015, pp. 293-297, China, September 2015.

[23] A. M. Hamed and R. K. Rao, "Energy and spectral efficiency in cellular networks considering fading, path loss, and interference," in Proceedings of the 30th IEEE Canadian Conference on Electrical and Computer Engineering, CCECE 2017, Canada, May 2017.

[24] H. Pervaiz, L. Musavian, and Q. Ni, "Area energy and area spectrum efficiency trade-off in $5 \mathrm{G}$ heterogeneous networks," in Proceedings of the IEEE International Conference on Communication Workshop, ICCW 2015, pp. 1178-1183, UK, June 2015.

[25] M.-S. Alouini and A. J. Goldsmith, "Area spectral efficiency of cellular mobile radio systems," IEEE Transactions on Vehicular Technology, vol. 48, no. 4, pp. 1047-1066, 1999.

[26] A. Mesodiakaki, F. Adelantado, L. Alonso, M. Di Renzo, and C. Verikoukis, "Energy-and Spectrum-Efficient User Association in Millimeter-Wave Backhaul Small-Cell Networks," IEEE Transactions on Vehicular Technology, vol. 66, no. 2, pp. 18101821, 2017.

[27] J.-M. Gorce, R. Zhang, K. Jaffrès-Runser, and C. Goursaud, "Energy, latency and capacity trade-offs in wireless multi-hop networks," in Proceedings of the 2010 IEEE 21st International Symposium on Personal Indoor and Mobile Radio Communications, PIMRC 2010, pp. 2757-2762, Turkey, September 2010. 


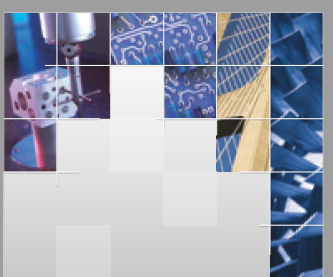

\section{Enfincering}
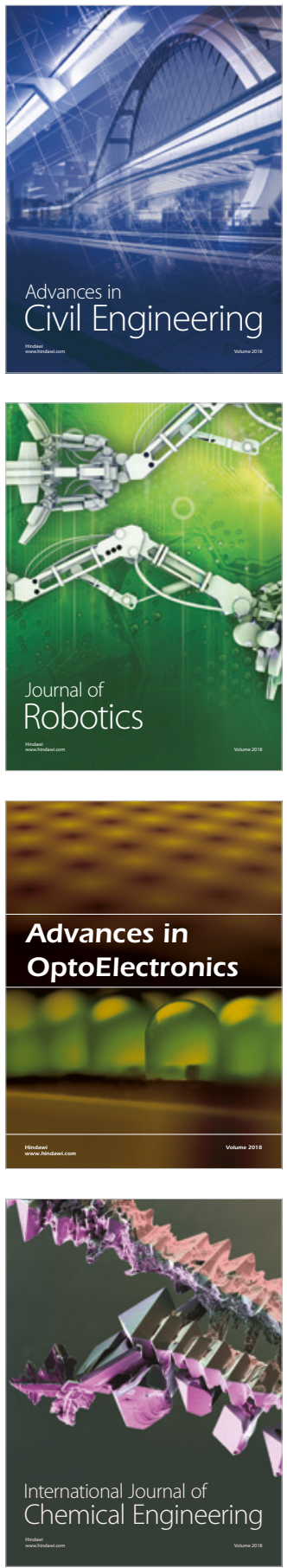

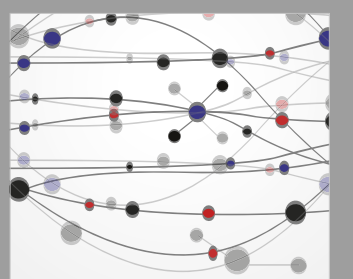

\section{Rotating \\ Machinery}

The Scientific World Journal

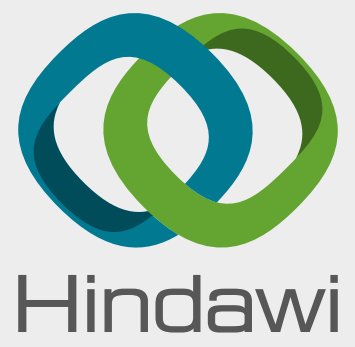

Submit your manuscripts at

www.hindawi.com
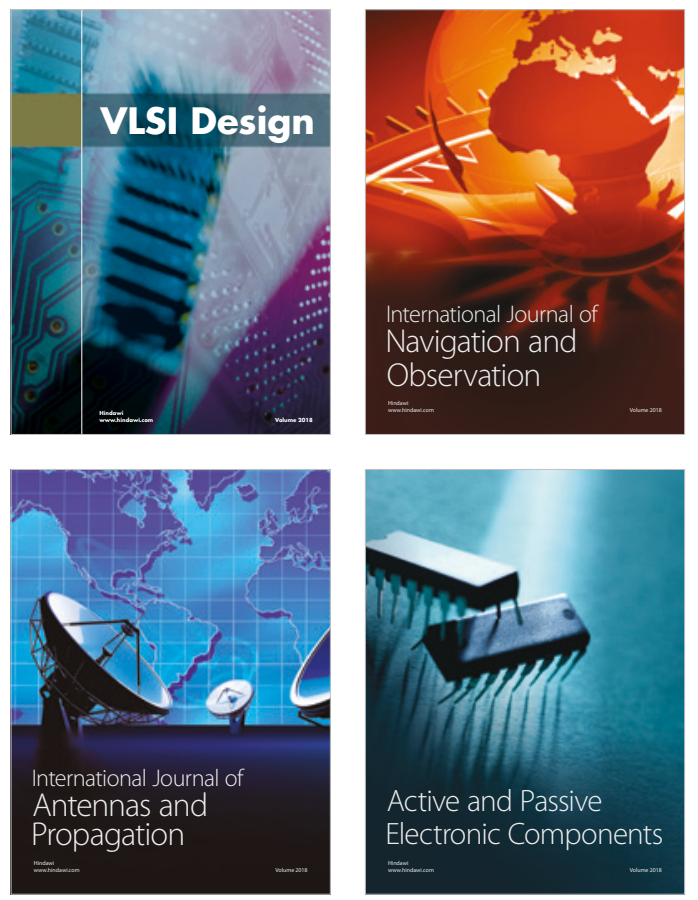
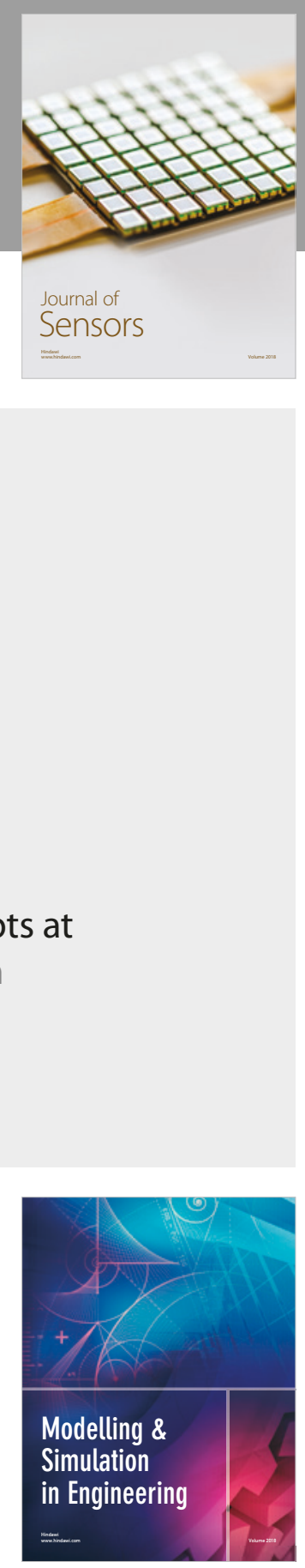

\section{Advances \\ Multimedia}
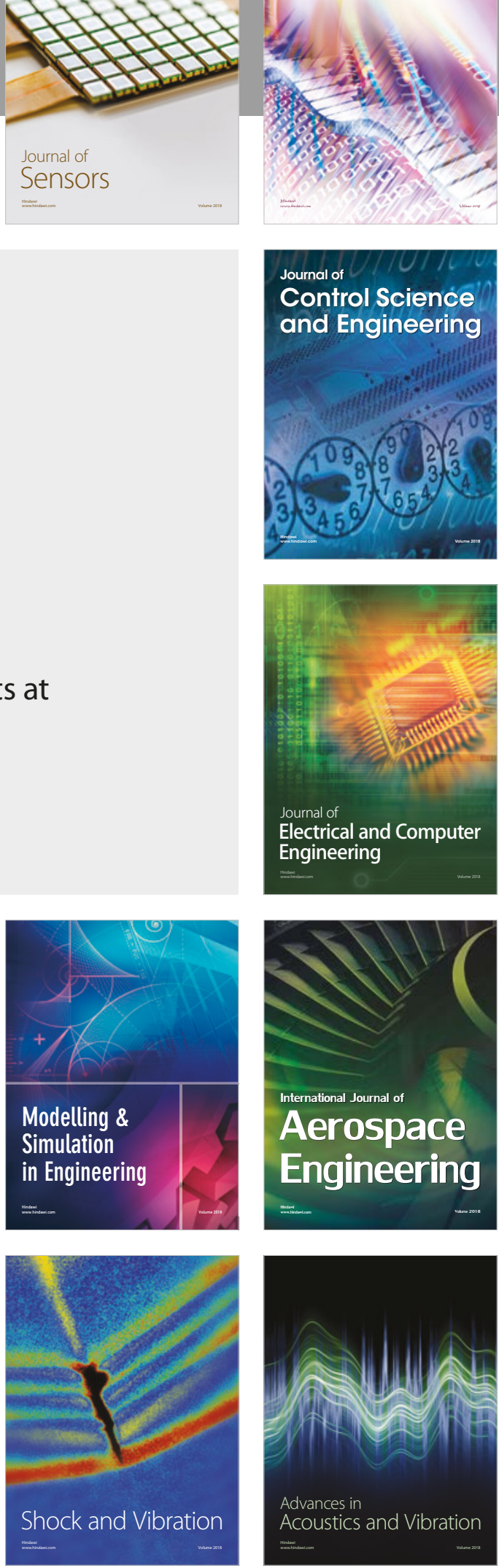